BMC

Veterinary Research

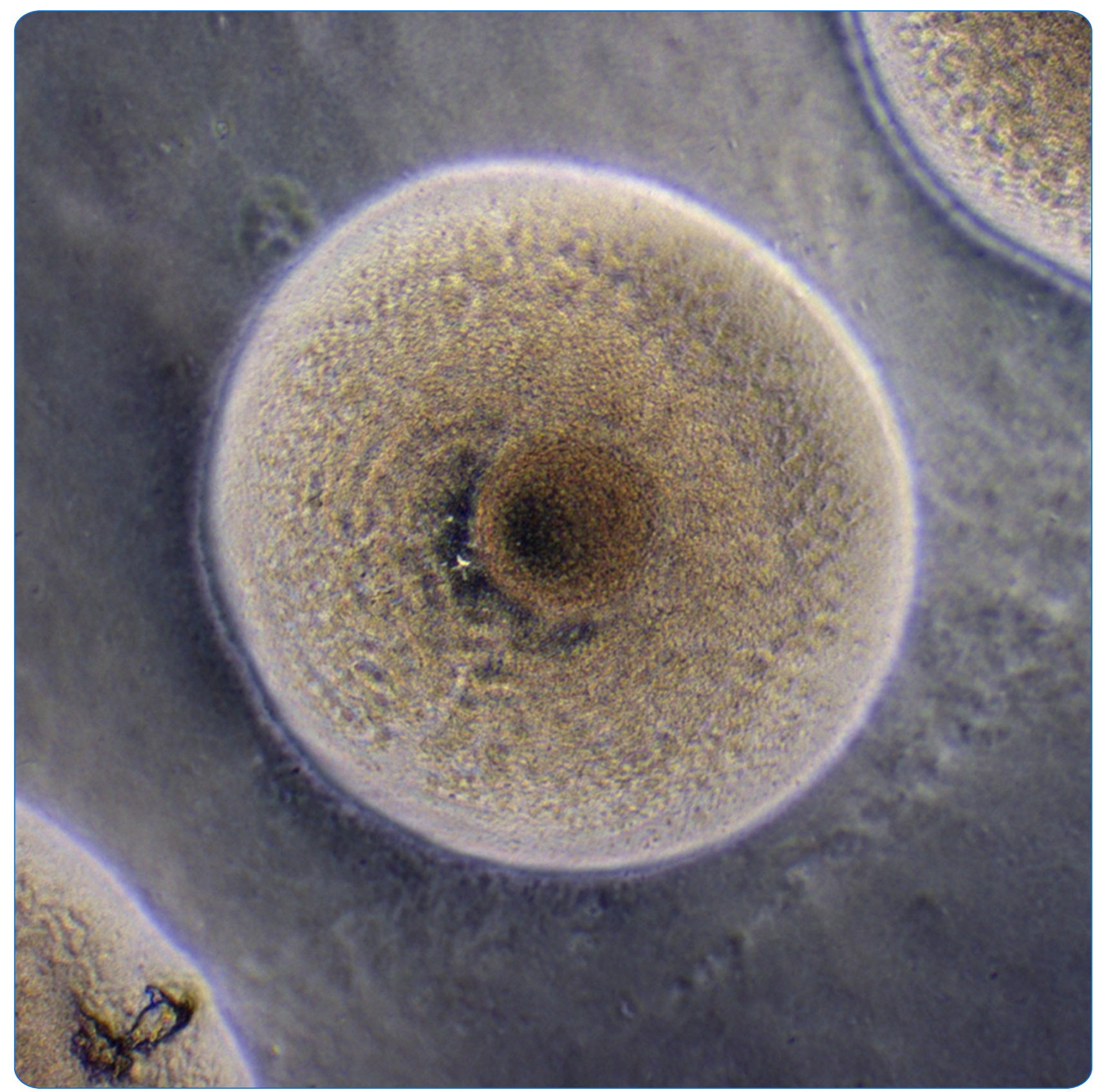

\title{
A survey of Mycoplasma agalactiae in dairy sheep farms in Spain
}

Ariza-Miguel et al. 


\title{
A survey of Mycoplasma agalactiae in dairy sheep farms in Spain
}

Jaime Ariza-Miguel, David Rodríguez-Lázaro* and Marta Hernández*

\begin{abstract}
Background: Contagious Agalactia (CA) is one of the major animal health problems in small ruminants because of its economic significance. Currently, four Mycoplasma spp. have been associated with this syndrome: M. agalactiae, M. mycoides subsp. capri, M. capricolum subsp. capricolum and M. putrefaciens. Their presence has been evaluated in several studies conducted in CA-endemic countries. However, previous Spanish studies have been focused on caprine CA, and there is a knowledge gap regarding which Mycoplasma species are present in sheep flocks from Spain, which has the second highest number of sheep amongst the 27 European Union member states.

Consequently, we investigated the presence and geographic distribution of the four CA-causing mycoplasmas in Spanish dairy sheep farms. This is the first time such an investigation has been performed.

Results: Three hundred thirty nine out of 922 sheep flocks were positive for M. agalactiae by real time PCR (36.8\%) and 85 by microbiological identification (9.2\%). Interestingly, all 597 milk samples assessed for the presence of M. mycoides subsp. capri, M. capricolum subsp. capricolum and M. putrefaciens tested negative. To evaluate the intermittent excretion of the pathogen in milk, we sampled 391 additional farms from 2 to 5 times, resulting that in $26.3 \%$ of the cases a previously positive farm tested negative in a later sampling.

Conclusions: M. agalactiae was the only Mycoplasma species detected in the study area showing a high frequency of presence and wide distribution. Therefore, the establishment of a permanent surveillance network is advantageous, as well as the implementation of control and prevention measures to hinder the dissemination of M. agalactiae and to prevent the entrance of other Mycoplasma species.
\end{abstract}

Keywords: Mycoplasma agalactiae, Contagious agalactia, Real time PCR, Sheep, Dairy, Spain

\section{Background}

Contagious Agalactia (CA) is, along with bovine pleuropneumonia and contagious caprine pleuropneumonia, one of the three Mycoplasma-induced diseases affecting small ruminants which are notifiable to the World Organisation for Animal Health due to their economic significance [1]. CA is a syndrome clinically characterized by mastitis, arthritis, keratoconjunctivitis and occasionally abortion [2,3] and Mycoplasma agalactiae is considered its major etiological agent. M. mycoides subsp. capri, M. capricolum subsp. capricolum and M. putrefaciens cause a clinically similar syndrome, particularly in goats $[4,5]$. The syndrome causes major economic losses because of reduction or suppression of milk production,

\footnotetext{
*Correspondence: ita-rodlazda@itacyl.es; ita-herperma@itacyl.es Instituto Tecnológico Agrario de Castilla y León (ITACyL), Consejería de Agricultura y Ganadería. Junta de Castilla y León, Carretera de Burgos km. 119, C.P. 47071, Valladolid, Spain
}

(c) 2012 Ariza-Miguel et al.; licensee BioMed Central Ltd. This is an Open Access article distributed under the terms of the Creative Commons Attribution License (http://creativecommons.org/licenses/by/2.0), which permits unrestricted use, distribution, and reproduction in any medium, provided the original work is properly cited.

abortion, high morbidity and mortality rates, and costs associated with the diagnosis, treatment and prevention which are estimated to be above 20 million Euros per year in the European countries forming the Mediterranean Basin $[3,4,6]$.

CA has its major impact in the Mediterranean countries, where the disease is considered to be endemic. However, it is also widely distributed in west Asian countries, central, north and east African Countries, the USA, and Brazil [1,2,7-9]. Interestingly, the significance of the different Mycoplasma species causing CA varies depending on the geographic area. In the United States, M. mycoides subsp. capri is the most prevalent caprine Mycoplasma, although $M$. agalactiae has been recently isolated [9]. In Northern Jordan, M. agalactiae and $M$. mycoides subsp. capri play the major role in both, sheep and goats $[7,10]$. In France, M. agalactiae has reemerged in sheep flocks located in the basin of the Western 
Pyrénées, causing 98 new outbreaks in 2008 [1]. In Spain, which has the second highest number of sheep amongst the 27 European Union member states, research has been focused on caprine CA, and there is a knowledge gap regarding which Mycoplasma species are present in sheep flocks from that country.

Consequently, in the present study we aimed to assess for the first time the presence and geographic distribution of the four Mycoplasma species causing CA, by analyzing raw milk samples from Spanish dairy sheep farms by classical microbiological methods, and PCRbased methods which have been demonstrated to be specific and sensitive [11-13]. The knowledge acquired will allow the implementation of appropriate control programs for those pathogens.

\section{Results}

Sensitivity of the PCR-based methods and capability to detect coinfected samples

Artificially contaminated milk samples $(50 \mathrm{~mL})$ inoculated with from $10^{8}$ cells to $10^{2}$ cells of each Mycoplasma gave positive results by the three PCR-based systems [1113]. Detection failed in the milk samples seeded with $10^{1}$ cells $(0.2$ cells $/ \mathrm{mL})$. Capability to detect samples coinfected with various CA-causing mycoplasmas was demonstrated in samples artificially contaminated with the 4 Mycoplasma species analyzed in this study.

\section{Detection of CA-causing mycoplasmas in Spanish sheep farms}

All 597 milk samples tested negative for $M$. mycoides subsp. capri, $M$. capricolum subsp. capricolum and $M$. putrefaciens. On the other hand, 339 out of 922 dairy sheep farms were positive for $M$. agalactiae by real time PCR (36.8\%). Furthermore, the pathogen was observed by microscopy identification in samples collected from 85 flocks (9.2\%). Thereby, 411M. agalactiae isolates were obtained.

We assessed the level of intermittent excretion of $M$. agalactiae during the sampling period using data obtained from other additional 391 farms sampled repeatedly from 2 to 5 times. In $26.3 \%$ of the cases a previously positive farm resulted negative in a later sampling and conversely, 38 farms (9.7\%) tested negative in the first sampling but resulted positive in any of the subsequent samplings. Overall, 250 of those sheep farms (63.9\%) were positive for M. agalactiae.

\section{Geographic distribution of flocks infected with Mycoplasma agalactiae}

Positive farms for $M$. agalactiae were located in all eleven provinces sampled (Figures 1, 2A). The frequency ranged from $7.7 \%$ to $100 \%$ of the flocks sampled per province revealing that the microorganism is widely distributed.

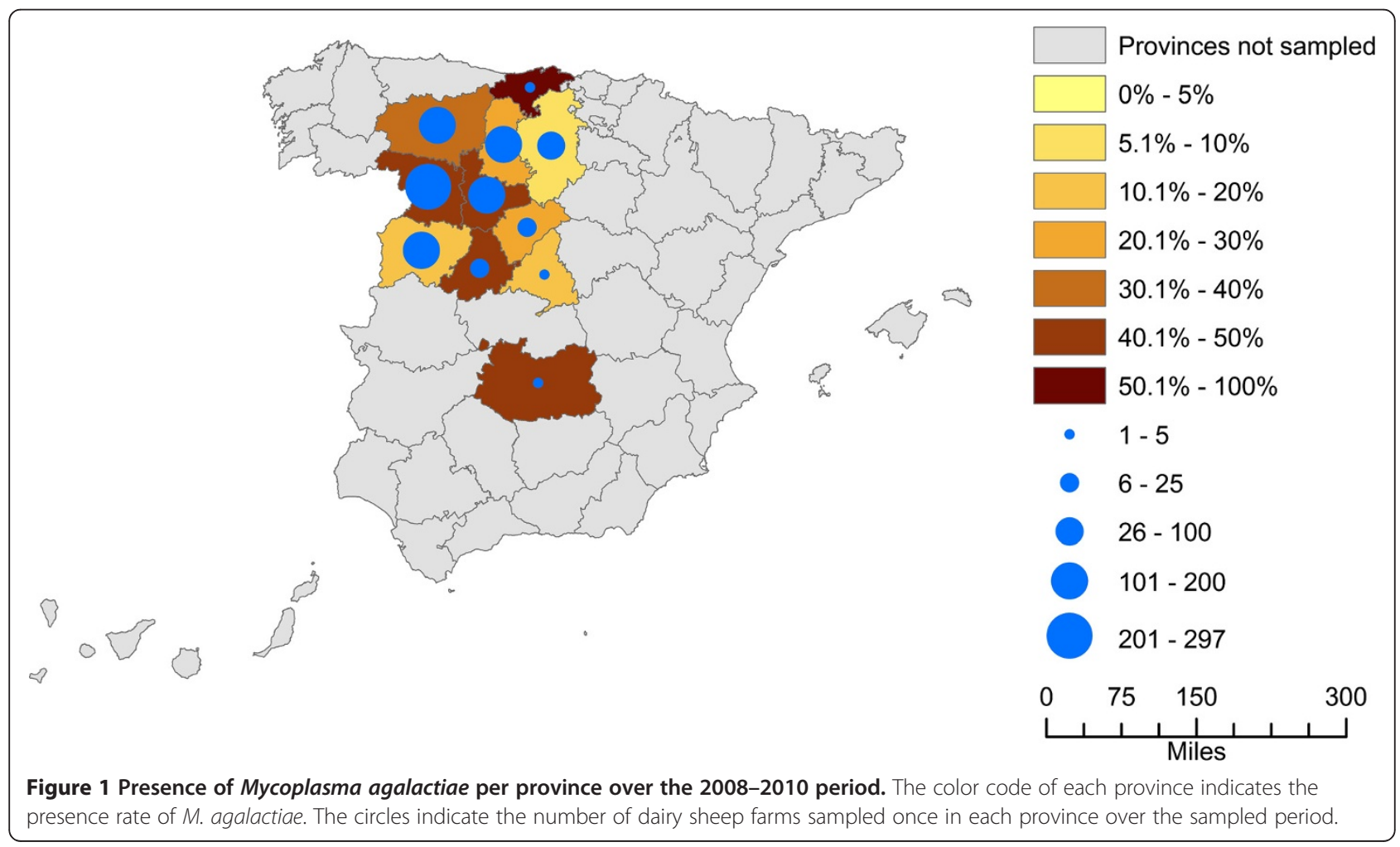




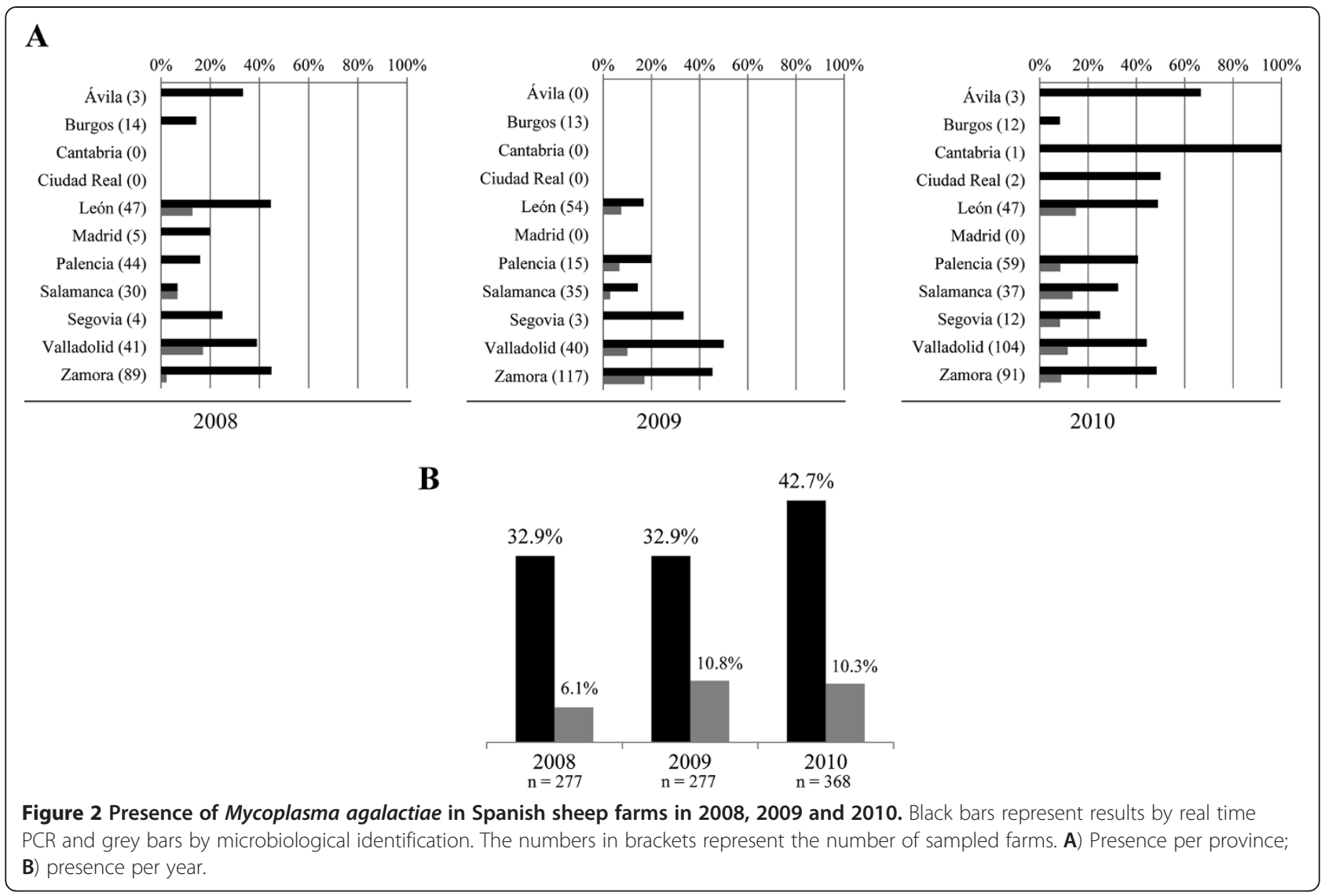

\section{Temporal distribution of Mycoplasma agalactiae}

The presence of $M$. agalactiae increased between 2008 (32.9\%) and 2010 (42.7\%) (Figure 2B). The minimum and maximum frequency of detection of $M$. agalactiae ranged from $7.7 \%$ in February 2009 to $53.1 \%$ in May 2009. The months when the pathogen was most frequently detected were May 2009 (53.1\%), July 2010 (51\%) and October 2008 (50\%). On the contrary, the lowest detection occurred in February 2009 (7.7\%), June 2009 (14\%) and April 2010 (16.7\%). Thereby, 72 out of 194 farms (37.1\%) were positive to $M$. agalactiae in Spring (i.e. farms analyzed in March, April and May), 113 out of $286(39.5 \%)$ in Summer (i.e. June, July and August), 110 out of 280 (39.3\%) in Autumn (i.e. September, October and November) and 44 out of 162 (27.2\%) in Winter (i.e. December, January and February).

\section{Discussion}

M. agalactiae was the only CA-causing Mycoplasma detected in dairy sheep farms located in the study area, and it was found to be widely distributed as it was detected in all eleven provinces sampled (Figure 1). In contrast, although $M$. agalactiae has been detected with a high prevalence in Spanish goat herds ranging from $40 \%$ to $66 \%[14,15]$, it seems that $M$. mycoides subsp. capri plays the major role in goat $[2,16]$. Interestingly, $M$. capricolum subsp. capricolum and $M$. putrefaciens have been recently isolated in goat herds located in the Canary Islands and Extremadura (southwestern Spain) regions $[17,18]$, and yet those pathogens have not been detected in sheep flocks from the study area suggesting that Spanish dairy sheep are free of their presence or, at least, that their distribution would be restricted outside the sampling area. Furthermore, in this study we have observed that the frequency of detection of $M$. agalactiae increased from $32.9 \%$ in 2008 to $42.7 \%$ in 2010, suggesting that the pathogen has spread in the sampling area during the study period. On the other hand, we tried to establish relationships between the excretion of the microorganism in milk and the stages in sheep milk production (e.g. period of parturitions, the start of lactation) by analyzing the presence of $M$. agalactiae per month and per season in the different sampling years, but no pattern was found. We hypothesize that this is because the farms from the sampled region frequently had different production systems, and the same production stage may have occurred in a different time between farms.

To our knowledge, few surveys have been carried out internationally to determine which Mycoplasma species 
are circulating in sheep. In Northern Jordan, investigations revealed a seroprevalence in sheep, goat and mixed flocks of $25 \%, 21 \%$ and $30 \%$ respectively, for $M$. agalactiae, and $32 \%$, 38\% and $34 \%$ for $M$. mycoides subsp. capri, suggesting that both microorganisms are widely distributed in that country and to the same extent in sheep and goats $[7,10]$. This scenario is probably caused because in Jordan more than $93 \%$ of sheep and goats are kept together in mixed farms, so both hosts are exposed to the same pathogens. In contrast, the existence of mixed farms in Spain is not common, hindering the dissemination of other Mycoplasma species from goat herds to sheep flocks.

Detection of $M$. agalactiae by using microbiological techniques $(9.2 \%)$ greatly underestimated its presence in comparison to detection by using real time PCR (36.8\%). This finding prompts the recommendation that PCRbased methods should be routinely used in Mycoplasma detection, as microbiological identification leads to underestimation due to the fastidious growth requirements of mycoplasmas, and serology is not a suitable method in those areas, such as Spain, where systematic vaccination is extended.

The results of this study require careful interpretation. Firstly, this syndrome follows a chronic course in endemic areas and the animals present an intermittent excretion of the microorganism in milk $[3,5]$. Therefore, the presence of $M$. agalactiae could have been underestimated, as samples were collected from the milk tanks. In fact, the findings obtained in this study suggest a high level of intermittent excretion, as in more than $26 \%$ of the re-sampled farms a previously positive flock tested negative in a later sampling and $63.9 \%$ overall tested positive. Considering that M. agalactiae is a highly persistent pathogen, remaining in the animals for years $[4,5]$, and that it is very difficult to eliminate from infected herds, we can assume that in most of those cases infected animals were not excreting the pathogen at the sampling time or that the number of bacteria present was below the limit of detection. Consequently, if monitoring of a herd needs be implemented, the inclusion of other suitable type of samples (e.g. blood, articular, auricular, ear canal, eye and vaginal swabs, nasal secretions, joint fluids) is strongly recommended. Secondly, the lower sensitivity of the conventional-PCR technique in comparison to the real time PCR could have underestimated the presence of the Mycoplasma species detected by the first method. Notwithstanding, all CA-causing mycoplasmas were detected after the inoculation of from $10^{8}$ to $10^{2}$ cells of each Mycoplasma spp. in $50 \mathrm{~mL}$ milk samples. Detection failed in samples containing $10^{1}$ cells $(0.2$ cells $/ \mathrm{mL})$ probably because the initial amount of mycoplasmas was not enough to compete during the enrichment step with all the other microbiota present in the milk samples. This suggests that all the PCR systems can detect the minimum quantity of mycoplasmas needed for growing in the enrichment step, and therefore that the presence of the Mycoplasma species as detected by conventional PCR would not be underestimated in detriment of the species detected by real time PCR. In addition, these results confirm that the enrichment step does not favor any species to the detriment to the others, and therefore that we can detect co-infected samples.

\section{Conclusions}

This study provides for the first time an overview of the presence of CA-causing mycoplasmas circulating in Spanish dairy sheep farms. M. agalactiae was the only species detected in the study area showing a high frequency of presence, and it was found to be widely distributed. The high frequency of detection of the pathogen supports the implementation of control and prevention measures to hinder the dissemination of $M$. agalactiae and to prevent the entrance of other Mycoplasma species, such as systematic laboratory detection, isolation or slaughtering of infected flocks and tracing of livestock exchange.

\section{Methods}

\section{Study area and sampling}

From August 2008 to July 2009, and from April to December 2010, 1,798 samples were taken from 1,313 dairy sheep farms located in twelve provinces: Ávila, Burgos, Cáceres, Cantabria, Ciudad Real, León, Madrid, Palencia, Salamanca, Segovia, Valladolid and Zamora. Most provinces belong to the Spanish region which presents the highest sheep milk production (60\% of total sheep milk) [19]. Selection of the farms was not performed following a formal randomization process, but blind samples were randomly provided by the Regional Interprofessional Dairy Laboratory. As blind sampling was carried out, 922 out of 1,313 farms were sampled once, and the resting 391 farms were sampled from 2 to 5 times. To ensure that no farms contribute more than once in the determination of the frequency of presence of the mycoplasmas, only those results obtained from the 922 farms sampled once have been used to perform the analyses showed in this study. The results obtained from the farms sampled several times have been only used to assess the level of intermittent excretion of the microorganisms in milk.

The sampling represents more than $16 \%$ of total dairy sheep farms in Spain and more than $44 \%$ of the farms located in the most productive region (Table 1). Location of the farms and overall number of dairy sheep per province is depicted in Figure 3. Each week, twenty raw milk samples of $50 \mathrm{ml}$ were taken from the refrigerated tanks 
Table 1 Geographic distribution of the Spanish dairy sheep farms sampled to detect CA-causing mycoplasmas

\begin{tabular}{lccc}
\hline Province & No. Farms & No. Sampled Farms & \% Sampled Farms \\
\hline Ávila & 91 & 9 & 9.9 \\
Burgos & 125 & 65 & 52.0 \\
Cáceres & 130 & 2 & 1.5 \\
Cantabria & 29 & 2 & 6.9 \\
Ciudad Real & 829 & 205 & 0.2 \\
León & 430 & 6 & 47.7 \\
Madrid & 174 & 177 & 3.4 \\
Palencia & 414 & 132 & 42.8 \\
Salamanca & 384 & 30 & 34.4 \\
Segovia & 79 & 292 & 38.0 \\
Valladolid & 491 & 391 & 59.5 \\
Zamora & 902 & $\mathbf{1 , 3 1 3}$ & 43.3 \\
12 provinces & $\mathbf{4 , 0 7 8}$ & $\mathbf{3 2 . 2}$ \\
\hline
\end{tabular}

Study areas for detecting the presence of CA-causing mycoplasmas in Spanish dairy sheep farms. The samples were collected from flocks located in twelve provinces showing highest sheep milk production in Spain.

and preserved during transportation at $4^{\circ} \mathrm{C}$ with $133 \mu \mathrm{L}$ of a bacteriostatic agent $\left(9.975 \times 10^{-2} \mu \mathrm{g}\right.$ chloramphenicol, 2.394 $\mu \mathrm{g}$ sodium azide, $1.33 \mu \mathrm{l}$ ethanol, $5.985 \mu \mathrm{g}$ trisodium citrate hydrate, $4.655 \times 10^{-2} \mu \mathrm{g}$ bromophenol blue). The samples were immediately analyzed because freezing considerably reduces the viability of mycoplasmas $[20]$.

\section{Culture of milk samples and DNA extraction}

One hundred microlitres of the milk samples were cultivated in $9.9 \mathrm{ml}$ of Mycoplasma broth base with Mycoplasma supplement G (Oxoid, Hampshire, UK), at $37^{\circ} \mathrm{C}$ in $5 \% \mathrm{CO}_{2}$ atmosphere for 3 days. For DNA extraction, $1 \mathrm{ml}$ of culture was centrifuged at $13,000 \times g$ for $15 \mathrm{~min}$, and the cell pellet was resuspended in $100 \mu \mathrm{l}$ of $10 \mathrm{mM}$ Tris $-\mathrm{HCl}\left(\mathrm{pH}\right.$ 8.0) for further incubation at $95^{\circ} \mathrm{C}$ with shaking for $20 \mathrm{~min}$. Then, samples were centrifuged at maximum speed for $3 \mathrm{~min}$, the supernatant was transferred into new tubes, and subsequently analyzed for CA-causing mycoplasmas.

\section{Molecular detection of CA-causing mycoplasmas by PCR-based methods}

Since $M$. agalactiae is the main aetiological agent, its presence was assessed in all farms. The presence of $M$. mycoides subsp. capri, M. capricolum subsp. capricolum and M. putrefaciens was evaluated in 597 samples taken from 528 sheep farms distributed in the eleven provinces. The latter samples were collected in July 2009 and since April to December 2010. Molecular detection of $M$. agalactiae was conducted by real time PCR as previously described [11] in a 7500 real time PCR system platform (Applied Bios stems, Carlsbad, CA, USA). $M$. mycoides cluster and M. putrefaciens detection was carried out by conventional PCR $[12,13]$. PCR amplification products were resolved by gel electrophoresis on $1.5 \%$ $(\mathrm{w} / \mathrm{v})$ agarose gels and visualized after staining with

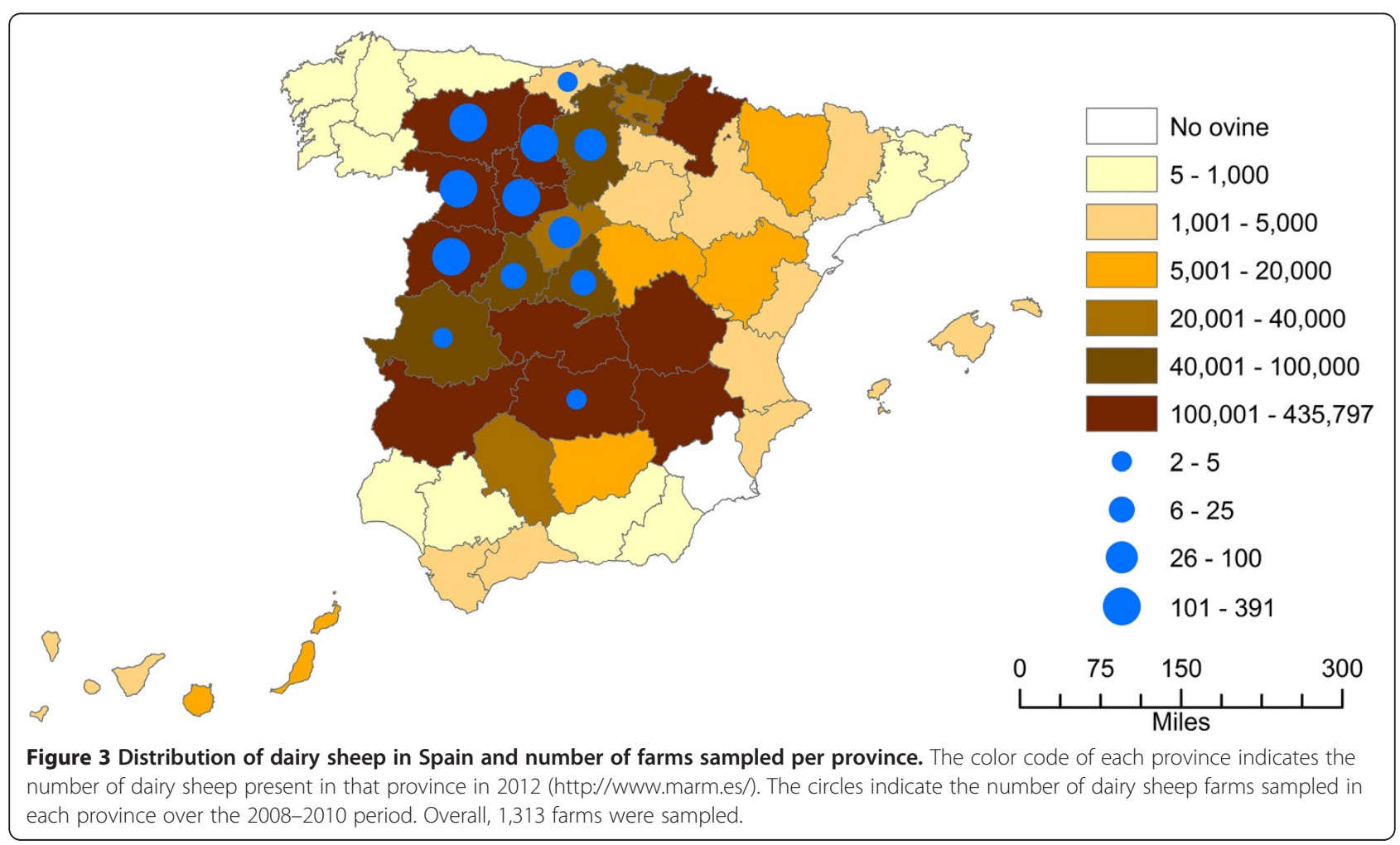


ethidium bromide by using a UV transilluminator (BioRad, Hercules, CA, USA). All samples were analyzed in duplicate and positive, negative extraction and non template controls were included in each run. The limit of detection of the real time PCR system corresponds to approximately 1 genomic equivalent per reaction. Those of the conventional PCR systems have not been reported. Therefore, in order to demonstrate that the PCR methods used in this study could detect the same levels of Mycoplasma contamination, and that detection of naturally co-infected samples would be possible, raw milk samples were artificially contaminated with decreasing concentrations of all four mycoplasmas and then processed following the protocol detailed above.

\section{Microbiological detection}

Pre-enriched milk samples were cultured in agar plates of the selective medium, which were incubated at $37^{\circ} \mathrm{C}$ in a $5 \% \mathrm{CO}_{2}$ atmosphere for 3 to 4 days. After incubation, colonies consistent with Mycoplasma phenotype were identified using an optical microscope Leica DMLS under $100 \times$ magnifications (LeicaMicrosystems $\mathrm{GmbH}$, Wetzlar, Germany).

\section{Isolation of mycoplasmas}

We isolated from 1 to 6 colonies from each positive milk sample and subculture them in specific broth medium at $37^{\circ} \mathrm{C}$, in a $5 \% \mathrm{CO}_{2}$ atmosphere for 3 days. The nature of the isolates was reconfirmed by real time PCR [11] and the purity of the culture by checking the morphology of the colonies on agar plates of the selective medium. Finally, the isolates were cryopreserved in glycerol $(16 \% \mathrm{v} / \mathrm{v})$ and stored at $-80^{\circ} \mathrm{C}$.

\section{Abbreviations \\ CA, Contagious Agalactia.}

\section{Competing interests}

The authors declare that they have no competing interests.

\section{Authors' contributions}

JA carried out the experiments, results analysis and drafted the manuscript. $\mathrm{DRL}$ and $\mathrm{MH}$ led the project, designed the study and revised the results and manuscript. All authors read and approved the final manuscript.

\section{Acknowledgements}

This study was supported by project RTA 2008-073 of the Spanish Ministry of Education and Science, Government of Spain. We thank Dr. Nigel Cook (FERA, UK) for critical revision of the manuscript.

Received: 3 July 2012 Accepted: 10 September 2012

Published: 24 September 2012

\section{References}

1. Chazel M, Tardy F, Le Grand D, Calavas D, Poumarat F: Mycoplasmoses of ruminants in France: recent data from the national surveillance network. BMC Vet Res 2000, 6:32.

2. De la Fe C, Assunção P, Antunes T, Rosales RS, Poveda JB: Microbiological survey for Mycoplasma spp. in a contagious agalactia endemic area. Vet J 2005, 170(2):257-259.
3. Lambert M: Contagious agalactia of sheep and goats. Rev Sci Tech 1987, 6(3):699-711.

4. Bergonier D, Berthelot X, Poumarat F: Contagious agalactia of small ruminants: current knowledge concerning epidemiology, diagnosis and control. Rev Sci Tech 1997, 16(3):848-873.

5. Corrales JC, Esnal A, De la Fe C, Sánchez A, Assunçao P, Poveda JB, Contreras A: Contagious agalactia in small ruminants. Small Rumin Res 2007, 68(1):154-166.

6. Nicholas RAJ: Improvements in the diagnosis and control of diseases of small ruminants caused by mycoplasmas. Small Rumin Res 2002, 45:145-149.

7. Al-Momani W, Abo-Shehada MN, Nicholas RAJ: Seroprevalence of and risk factors for Mycoplama mycoides subspecies capri infection in small ruminants in Northern Jordan. Trop Anim Health Prod 2011, 43(2):463-469.

8. De Azevedo EO, De Alcântara MDB, Do Nascimento ER, Tabosa IM, Barreto ML, De Almeida JF, Araújo MD, Rodrigues ARO, Riet-Correa F, De Castro RS: Contagious Agalactia by Mycoplasma agalactiae in small ruminants in Brazil: first report. Braz J Microbio/ 2006, 37(4):576-581.

9. Kinde H, DaMassa AJ, Wakenell PS, Petty R: Mycoplasma infection in a commercial goat dairy caused by Mycoplasma agalactiae and Mycoplasma mycoides subsp. mycoides (caprine biotype). J Vet Diagn Invest 1994, 6(4):423-427.

10. Al-Momani W, Nicholas RAJ, Abo-Shehada MN: Risk factors associated with Mycoplasma agalactiae infection of small ruminants in northern Jordan. Prev Vet Med 2008, 83:1-10.

11. Oravcová K, López-Enríquez L, Rodríquez-Lázaro D, Hernández M: Mycoplasma agalactiae $p 40$ Gene, a novel marker for diagnosis of Contagious Agalactia in sheep by Real-Time PCR: assessment of analytical performance and in-house validation using naturally contaminated milk samples. J Clin Microbiol 2009, 47(2):445-450.

12. Peyraud A, Woubit S, Poveda JB, De la Fe C, Mercier P, Thiaucourt F: A specific PCR for the detection of Mycoplasma putrefaciens, one of the agents of the contagious agalactia syndrome in goats. Mol Cell Probes 2003, 17(6):289-294.

13. Woubit S, Manso-Silván L, Lorenzon S, Gaurivaud P, Poumarat F, Pellet M-P, Singh VP, Thiaucourt F: A PCR for the detection of mycoplasmas belonging to the Mycoplasma mycoides cluster: Application to the diagnosis of contagious agalactia. Mol Cell Probes 2007, 21(5-6):391-399.

14. Andrada M, Déniz S, González M, Rodríguez F, Poveda JB: Epidemiology of Mycoplasma mycoides subsp. mycoides LC in caprine herds by indirect ELISA in Gran Canaria: preliminary results. In COST 826. Agriculture and Biotechnology: Mycoplasmas of Ruminants: Pathogenicity, Diagnostics. Edited by Poveda J, Fernandez A, Frey J, Johansson KE. Brussels: Epidemiology and Molecular Genetics, vol.5. European Communities; 2000:126-129.

15. Assunçao P, De la Fe C, Ramirez AS, Andrada M, Poveda JB: Serological study of contagious agalactia in herds of goats in the Canary Islands. Vet Rec 2004, 154:684-687.

16. Amores J, Gómez-Martín A, Corrales JC, Sánchez A, Contreras A, De la Fe C Presence of contagious agalactia causing mycoplasmas in Spanish goat artificial insemination centres. Theriogenology 2011, 75(7):1265-1270.

17. De la Fe C, Gutierrez A, Poveda JB, Assunsao P, Ramírez AS, Fabelo F: First isolation of Mycoplasma capricolum subsp. capricolum, one of the causal agents of caprine contagious agalactia, on the island of Lanzarote (Spain). Vet J 2007, 173(2):440-442.

18. Gil MC, Peña FJ, Hermoso De Mendoza J, Gomez L: Genital Lesions in an Outbreak of Caprine Contagious Agalactia Caused by Mycoplasma agalactiae and Mycoplasma putrefaciens. J Vet Med 2003, 50(10):484-487.

19. The Spanish Ministry of Agriculture: Food and Environment (MARM). database [http://www.marm.es/app/vocwai/Inicio.aspx?sec=SOC].

20. Amores J, Sánchez A, Martín AG, Corrales JC, Contreras A, De La Fe C: Viability of Mycoplasma agalactiae and Mycoplasma mycoides subsp. capri in goat milk samples stored under different conditions. Vet Microbiol 2010, 145(3-4):347-350.

doi:10.1186/1746-6148-8-171

Cite this article as: Ariza-Miguel et al:: A survey of Mycoplasma agalactiae in dairy sheep farms in Spain. BMC Veterinary Research 2012 8:171. 